\title{
User Informatics Optimized Search and Retrieval-Congestion Avoidance Scheme for 4G Networks
}

\author{
Pushpa $^{1}$, Sweta Sneha ${ }^{2}$, Rajeev Agrawal ${ }^{3 *}$ \\ ${ }^{1}$ Department of Information Technology, Accurate Institute of Technology \& Management, Greater Noida, India \\ ${ }^{2}$ Department of Computer Science and Information System, Kennesaw State University, Kennesaw, USA \\ ${ }^{3}$ Department of Computer Science, G. L. Bajaj Institute of Technology \& Management, Greater Noida, India \\ Email: pushpa_gla@yahoo.co.in, ssneha@kennesaw.edu, rrajkecd@gmail.com
}

Received March 10, 2012; revised April 13, 2012; accepted May 11, 2012

\begin{abstract}
The objective of $4 \mathrm{G}$ network is to provide best services to the users which in turn made the performance of existing network more critical. Further, the large traffic generated in such networks creates congestion resulting in overloading of the system. Frequent delays, loss of packets, and in addition the number of retransmission/paging also increases the computational cost of the system. This paper proposes a novel way to reduce overloading and retrieval mechanism for VLR through optimized search, based on the information of users mobility pattern (User profiles based (UPB)) to track the user. This not only improves the overall performance of the system, especially in the events when the visitor location register (VLR) is overloaded due to heavy traffic and congestion of the network. It was also established through simulation studies that the proposed UPB scheme optimizes the search and reduces the average waiting time in a queue. In addition, the provision of $V L R_{w}$ (waiting visitor location register) avoids the overloading of main VLR and provides a recovery/retrieval mechanism for VLR failure.
\end{abstract}

Keywords: Mobile Network; Optimization; Overloading; Location Management; User Profile

\section{Introduction}

Mobile cellular technology has evolved at a fast rate over the past few decades. In a cellular network, user mobility is considered as main phenomenon and also a major concern. A number of solutions are taken into consideration to provide seamless mobility support to the users irrespective of geographical conditions and network deployment. As a result of this, the accessible range has been increased in a reach from campuses to cites, countries or even continents. Such seamless mobility criteria poses a challenging issue among the designers, firstly, the designers must design system to adapt frequent changes in the topology in a transparent manner to the end user. The network is scalable to accommodate the large number of users which keep on adding to mobile network at a phenomenal rate. Therefore to maintain quality of service in such growing networks are always a challenge for the designers. In wireless networks, mobility support is one of the key features which enables a mobile user to communicate with another user regardless of its location. Location and resource management are the two main critical issues of any wireless network.

To better understand this process in cellular network

*Corresponding author.
Home Location Register (HLR) and Visitor Location Register (VLR) are two main database registers. HLR and VLR are used for storing location information of the users. Moreover a 2-tier system based on HLR and VLR databases are required to track the calls in the networks. Congestion in the network is judged by the arrival of customer and mean time spent in the network. Hence, one must obviate the failure or overloading of HLR and VLR to avoid any congestion in the networks. The proposed work computes the probability of VLR overloading based on the critical value defined for the overloading status of VLR, in case the threshold is achieved the other users are moved in waiting VLR $\left(V L R_{w}\right)$ to reduce the overloading of main VLR. However, $\left(V L R_{w}\right)$ is a substitute of VLR which is used to search the user in a network by employing a user profile based (UPB) scheme. It is important to note that Quality of Service (QoS) of a wireless network depends upon the two major functions viz efficiency of the system to locate the user and resource management. Therefore, it becomes important for location schemes to track the mobile user movement pattern accurately and within a specified time. Location management addresses this issues of how to track and locate a mobile user within a network as well as the use of HLR and VLR for location management [1,2]. 
However, with a 4G wireless support there will be several other issues in front of the researchers to be achieved taken into consideration the salient features of $4 \mathrm{G}$ i.e. a user centric approach through customization. Some of the key features of $4 \mathrm{G}$ networks via full packet switched network are anyone, anytime and anywhere communication, unlimited spectrum and its allocation, support for multimedia services at low bit transmission cost, increasing the user loyalty, proper utilization of radio resources and above all applications at minimum cost $[3,4]$. While the number of mobile subscribers is increasing, it is also important from a service providers point of view to have customer loyalty. This is one of the main objectives of service providers that lead to research how to increase the loyalty of mobile subscribers. Service providers are not only concentrating on increasing their subscribers but also want to retain them. To achieve these objective the quality of services plays a vital role and congestion avoidance by any means become inevitable.

This paper focuses on obtaining the probability of VLR overloading in conventional system and transferring mobile users in $V L R_{w}$ in case if VLR overloading. $V L R_{w}$ is a new database which is created on the basis of mobility prediction patterns of the user. To obtain this, we have used a new optimized search scheme known as UPB scheme for mobility prediction. In previous studies various algorithms or techniques have been used to predict the user mobility patterns $[5,6]$. However, the proposed scheme is based on the analysis of the user movement from one cell to another in different timestamp and representing it through a transition probability matrix (TPM). As it is evident that generally a user follows a fixed route or schedule in his day to day life so all the preferable location is generally known. This prior information is added to the profile of the user leading to a mechanism named as user profile based (UPB) scheme. The paper is organized in way that in Section 2, we have described the mobility database used in cellular networks. Section 3 outlines the VLR overloading state, Section 4 presents the proposed UPB scheme along with its comparative study with other search techniques. Followed by Sections 5 and 6 presenting the retrieval mechanism and criteria for moving the user in $V L R_{w}$. Finally, we conclude the outcome in Section 7.

\section{Mobility Database}

This section gives information about the database used in mobile networks. As discussed in previous section that in Wireless Cellular networks, there are two types of databases namely HLR and VLR. The HLR is a database that contains permanent mobile subscriber information. Also HLR consists of subscriber information, location information, service restrictions and supplementary services.
A VLR is a database which contains temporary information concerning the mobile subscribers that are currently located in a given MSC serving area but whose HLR is elsewhere. A subscriber cannot be present in more than one VLR at a time. The VLR is another location register used to retrieve information for calls to or from a visiting mobile user [1]. The following task is to be performed by VLR.

1) VLR informs the HLR that a subscriber has arrived in his area which is to be covered by the VLR.

2) VLR tracks the subscriber within the location area when there is no ongoing call.

3) VLR deletes the subscriber record when a subscriber explicitly moves to another cell as instructed by the HLR.

In this paper we have computed the VLR overloading and failure state and accordingly we have initiated a retrieval mechanism based on user profile based scheme to track the mobile user location during the following states 1) In case when VLR is overloaded; 2) In case of VLR Failure and 3) In case where there is a heavy congestion in the network which may lead to a deadlock situation. It is to be noted that if the location database fails due to any of the reasons, the information of the locations will be lost and the call will not be established. Such a scenario will result in the degradation of the quality of service offered by a particular service provider or network to subscribers. Having used one more VLR named as waiting VLR $\left(V L R_{w}\right)$ recovery mechanism will be triggered only during the situation when main VLR is overloaded or failed. The proposed scheme also computes VLR overloading state and determines the number of users who will be moved in $V L R_{w}$ and compares the performance of proposed scheme.

\section{Overloading State/Condition of VLR}

This section discusses the situation of VLR overloading. For this one has to compute the probability of the VLR overloading in case of a mobile network. Further, the proposed scheme in such kind of situation utilizes the services of $V L R_{w}$ to take over the workload of the failed or overloaded VLR. The performance evaluation of a $V L R_{w}$ is also carried out to analyze the functioning of $V L R_{w}$, the following assumptions/parameters are taken into consideration [7].

1) The arrivals of no. of query in VLR follow a Poisson distribution with the mean arrival rate $\lambda_{\alpha}$.

2) The mean time spend in a queue i.e. VLR is $1 / \mu_{r}$.

3) Maximum number of users associated with a VLR is $C$.

4) The failure rate of a VLR is $\lambda$, which gives the average number of failure occurring in VLR within a given time period. 
5) The recovery rate of a VLR is $\mu$, which is the average number of recoveries performed within a given time period.

6) The probability that a VLR is a case of overloading status is $P_{\text {overload }}$.

7) The probability that VLR is a case of failure status is $P_{\text {failure }}$.

8) The average movements of a failure-affected VLR user to be associated with a qualified backup $V L R_{w}$ are User $_{\text {moves }}$.

Based on the above assumption the $P_{\text {overload }}$ [7] can be expressed as:

$$
P_{\text {overload }}=\sum_{n=k}^{C} \frac{\frac{\left(\lambda_{a} / \mu_{r}\right)^{n}}{n !}}{\sum_{i=0}^{C} \frac{\left(\lambda_{a} / \mu_{r}\right)^{i}}{i !}}
$$

where $P_{\text {overload }}$ is the probability that if there are n number of VLR users within the coverage range of a VLR and the maximum number of users that can be associated with a VLR is $k$, then if the number of VLR user associated is greater than or equal to $k$, the VLR is regarded to be in the overloading states.

For similar cases the $P_{\text {failure }}$ [7] can be obtained based on the fourth and fifth parameter:

$$
\begin{aligned}
P_{\text {failure }} & =1-\frac{1}{1+\frac{\lambda}{\mu}} \\
& =\frac{\lambda}{\lambda+\mu}
\end{aligned}
$$

The term $\frac{\lambda}{\lambda+\mu}$ is the availability of VLR at a par- ticular instant of time.

To compute the probability of VLR overloading one has to use the following procedure/steps:

Step-1 for $\mathrm{n}=$ number of user (20): C

Step-2 for $\mathrm{I}=0$ : C

Step-3 $p_{1}=\frac{\left(\frac{\lambda_{a}}{\mu_{r}}\right)^{n}}{n !}, \quad p_{2}=\frac{\left(\frac{\lambda_{a}}{\mu_{r}}\right)^{i}}{i !}, \quad P_{\text {overload }}=\frac{p_{1}}{p_{2}}$

Step-4 if $\left(P_{\text {overload }}=\geq 1\right)$

Printf 'Now VLR is overloaded'

else

Printf 'Following $\mathrm{n}$ No. of users can be in probability of being overloaded'

Step-5 End

For better understanding of the overloading status of the VLR, we have plotted the value $P_{\text {overload }}$ for different values of $\lambda_{a}$ and $1 / \mu_{r}$ in Figure 1 and the percentage of overloading was noted with a fixed value of $C$ (in this case the value is taken as 20,000). Further, we have assumed that with overloading probability of 0.9 , the VLR will be in a failed state due to overloading and congestion of the network. Assume that there is only one VLR in the system. If $n=20$ thousands is maximum capacity of one VLR, then according to the above algorithm the following graph is obtained.

It was observed from the table that VLR overloading depends on the value of $\lambda_{a}$ and $1 / \mu_{r}$ Further; the values obtained from the plots are tabulated in Table 1. which indicates the number of users who will be under the influence of VLR overloading. For example in case 5, around nineteen thousands users have 0.6 probability of being overloaded in VLR whereas in case-2, nineteen

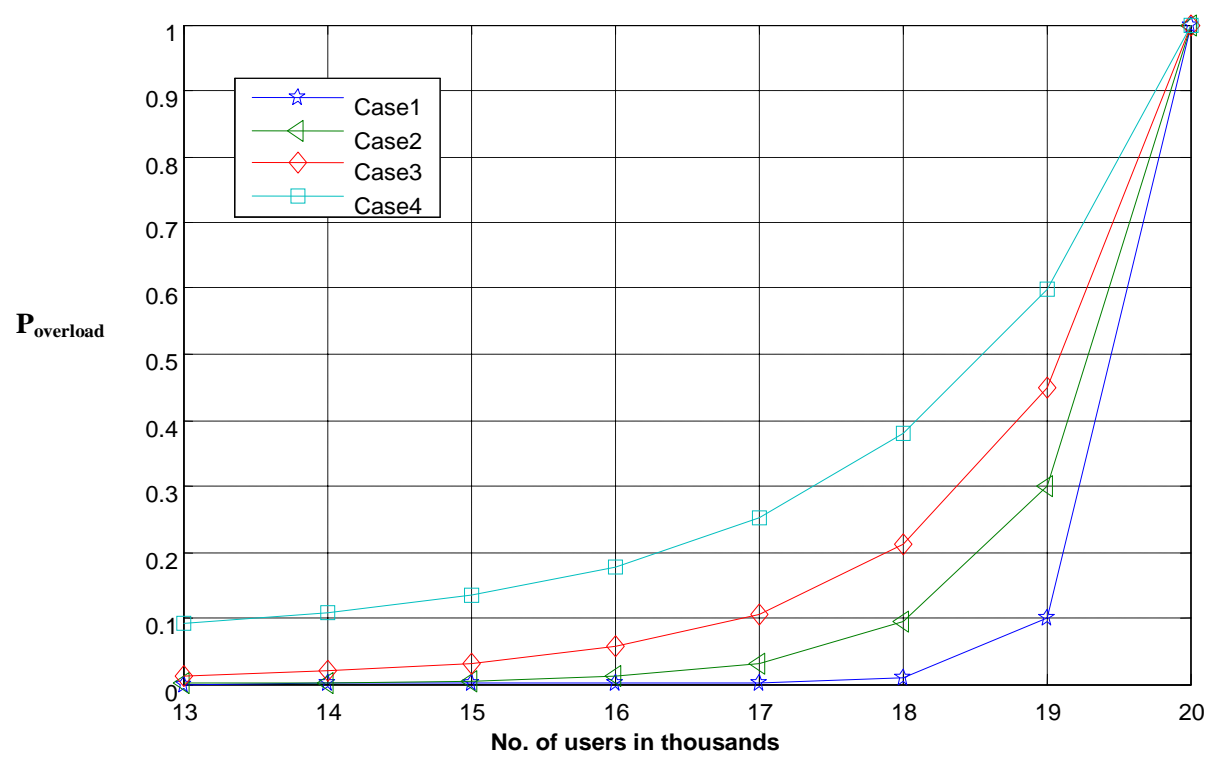

Figure 1. No. of users vs Probability of overloading for different cases. 
Table 1. Gives the status for probability of VLR failure for different values of parameters.

\begin{tabular}{ccccc}
\hline Case No. & $\begin{array}{c}\text { Number of arrival in VLR } \\
\left(\lambda_{a}\right) \text { per second }\end{array}$ & $\begin{array}{c}\text { Mean time spend in } \\
\text { VLR (In sec.) }\end{array}$ & $\begin{array}{c}\text { The probability of overloading } \\
\text { for 19,000 users }\end{array}$ & $\begin{array}{c}\text { Probability of } \\
\text { VLR failure }\end{array}$ \\
\hline 1 & 1 & 2 & 0.10 & 0.11 \\
2 & 3 & 2 & 0.30 & 0.33 \\
3 & 2 & 3 & 0.30 & 0.33 \\
4 & 3 & 3 & 0.45 & 0.50 \\
5 & 3 & 4 & 0.60 & 0.66 \\
\hline
\end{tabular}

thousand users have 0.3 probability of being overloaded. To establish the model suitability, we have taken the following value of the parameters to compute the probability of failure $\lambda=0.01, \mu=0.19$. As per Equation (2), one can obtain the $P_{\text {failure }}$ as

$$
P_{\text {failure }}=\frac{0.01}{0.01+0.190}=0.050
$$

However, it will be worth mentioning that if by any mean one can move certain number of users from VLR, the condition of overload will be avoided. Therefore it is suggested to move the users to $V L R_{w}$ in case of VLR overloading, this condition will depend on the probability of VLR failure, $\lambda_{a}$ and $1 / \mu_{r}$.

\section{Proposed User Profile Based (UPB) Scheme}

As discussed in the previous section whenever the VLR is in an overloading state, some of the users will be moved to $V L R_{w}$. It is important to note that in $V L R_{w}$ a new scheme for searching based on the information of the user's movement from one cell to another in different timestamp is used. Further, it is evident that in day to day routine the user generally follows a fixed route or schedule, so information on all such preferable locations of a particular user can be gathered. This prior information is added to the profile of a user and a new scheme proposed through UPB. Future positions must be predicted based on historical records and mobility related parameters such as direction, speed and current position [8-10].

The scheme utilizes three basic information of the user i.e. the user transition from one cell to another, construction of TPM based on the user transition and finally calculating the steady state values for each location [11]. For a better understanding of the proposed scheme, we have taken the following observations of a particular user in different time stamps:

$1 / 2 / 7 / 1 / 2 / 3 / 4 / 1 / 2 / 3 / 5 / 1 / 2 / 3 / 6 / 1 / 2 / 3 / 6 / 1 / 2 / 3 / 7 / 1 / 2 / 1 / 1 / 2 /$ $1 / 2 / 3 / 2 / 1 / 5 / 3 / 1 / 6 / 1 / 3 / 1 / 7 / 6 / 5 / 4 / 1 / 2 / 4 / 3 / 7 / 1 / 2 / 3 / 1 / 2 / 7 / 2 / 1 / 6$ /5/3/3/2/1/4/5/2/5/6/2/6/4/7/7/5/5/7/4/4/6/6/7/3/4/2/6/3/2/ 6 ... based on this information we have constructed a probability transition matrix, $P_{U P B}$ on the basis of the user transition.

$$
P_{U P B}=\left[\begin{array}{ccccccc}
1 / 18 & 12 / 18 & 1 / 18 & 1 / 18 & 1 / 18 & 1 / 18 & 1 / 18 \\
6 / 18 & 1 / 18 & 5 / 18 & 1 / 18 & 1 / 18 & 1 / 18 & 3 / 18 \\
4 / 14 & 5 / 14 & 1 / 14 & 1 / 14 & 1 / 14 & 1 / 14 & 1 / 14 \\
2 / 8 & 1 / 8 & 1 / 8 & 1 / 8 & 1 / 8 & 1 / 8 & 1 / 8 \\
1 / 8 & 1 / 8 & 1 / 8 & 1 / 8 & 2 / 8 & 1 / 8 & 1 / 8 \\
1 / 9 & 1 / 9 & 3 / 9 & 1 / 9 & 1 / 9 & 1 / 9 & 1 / 9 \\
1 / 9 & 1 / 9 & 3 / 9 & 1 / 9 & 1 / 9 & 1 / 9 & 1 / 9
\end{array}\right]
$$

Further, the $P_{U P B}$ is used to analyze most probable location of a user by calculating the steady state value $S_{U P B}$. This is a row vector consists of steady state for all seven cells. Each steady state represents the probability of users' most likelihood location. Index of the row vector represents cell number.

$$
\begin{aligned}
& S_{U P B} \\
& =[0.0532,0.0220,0.1217,0.3439,0.2945,0.1129,0.0518]
\end{aligned}
$$

It was observed that in row vector $S_{U P B}$, cell4 represents that most likelihood location of a user. Finally we add this information in mobile database to optimize the number of searches of a user. In case user1 calls to user2, the search will take place according to the maximum likelihood availability of location of user2 which follows the decreasing values of the $S_{U P B}$ row vector.

\section{Reduction of VLR Overloading}

It can be noticed that one can reduce the VLR overloading state by two ways i.e. first, by reducing searching time of location or through reducing average waiting time of mobile user in system and second, by reducing the number of users in the system. As discussed in the previous sections that UPB scheme is used to reduce the searching time of the caller. However, $P_{\text {overload }}$ depend on the mean time of user spends $1 / \mu_{r}$ in a system, $P_{\text {overload }}$ is directly proportional to $1 / \mu_{r}$.Consider the following row vector $S_{U P B}$ for steady state.

$$
\begin{aligned}
& S_{U P B} \\
& =[0.0531,0.0220,0.1217,0.3439,0.2945,0.1129,0.0518]
\end{aligned}
$$


The graph presented below indicates the number of searches required in UPB scheme.

It will be interesting to observe the performance of other searching schemes compared to UPB. The other schemes generally employed are:

\subsection{Random Search Technique (RST)}

In RST, user can be searched in any random order. We have taken any random pattern to search the user. Here we have taken the following order.

$$
V_{R S T}=[2,3,1,6,7,4,5]
$$

Row vector $V_{R S T}$ shows that search order is first cell2. If the user is present here, then call is transferred there otherwise search in cell3 and so on.

\subsection{Sequential Search Technique (SST)}

In SST, user can be searched in sequential order of cells, means in an increasing order of cells. Row vector shows that such order is cell1, cell2 ... cell7.

$$
V_{S S T}=[1,2,3,4,5,6,7]
$$

Comparing the different searching scheme it was clear in Figure 2 that UPB scheme can significantly reduce the searching time, as searching time is directly proportional to the number of searches required to search user location. Consider an example where a user is present in cell no. 4 and if we are searching according to UPB then it will take only 1 search, while 6 in RST and 4 in SST. We know that searching time is directly proportional to the no. of search.
Searching time $\propto$ No. of search

Searching time $=K *$ No. of search where

$K=$ Time required in one search.

Figure 3 shows total searching time in different three searching techniques such as UPB, RST and SST for different values of $K$. It is clear from Figure 3 that UPB performs well as compared to other schemes.

However RST and SST can also perform well in some cases but we cannot predict about those cases. As far as UPB is concerned, it performs well under all the conditions because UPB is based on the past history behavior of users [12]. Suitability of UPB scheme is on continuously analyzing the location behavior of the user.

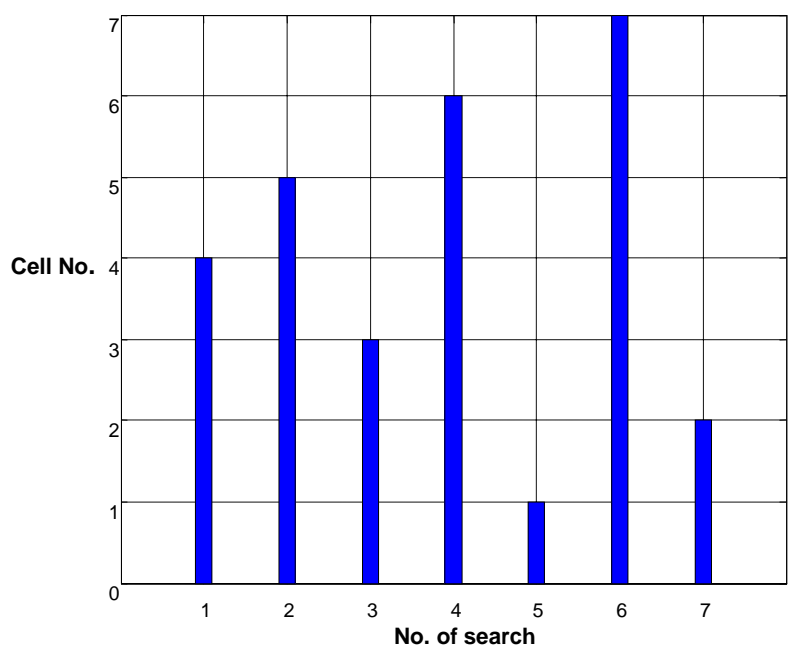

Figure 2. No. of searches vs Cell no. using UPB.

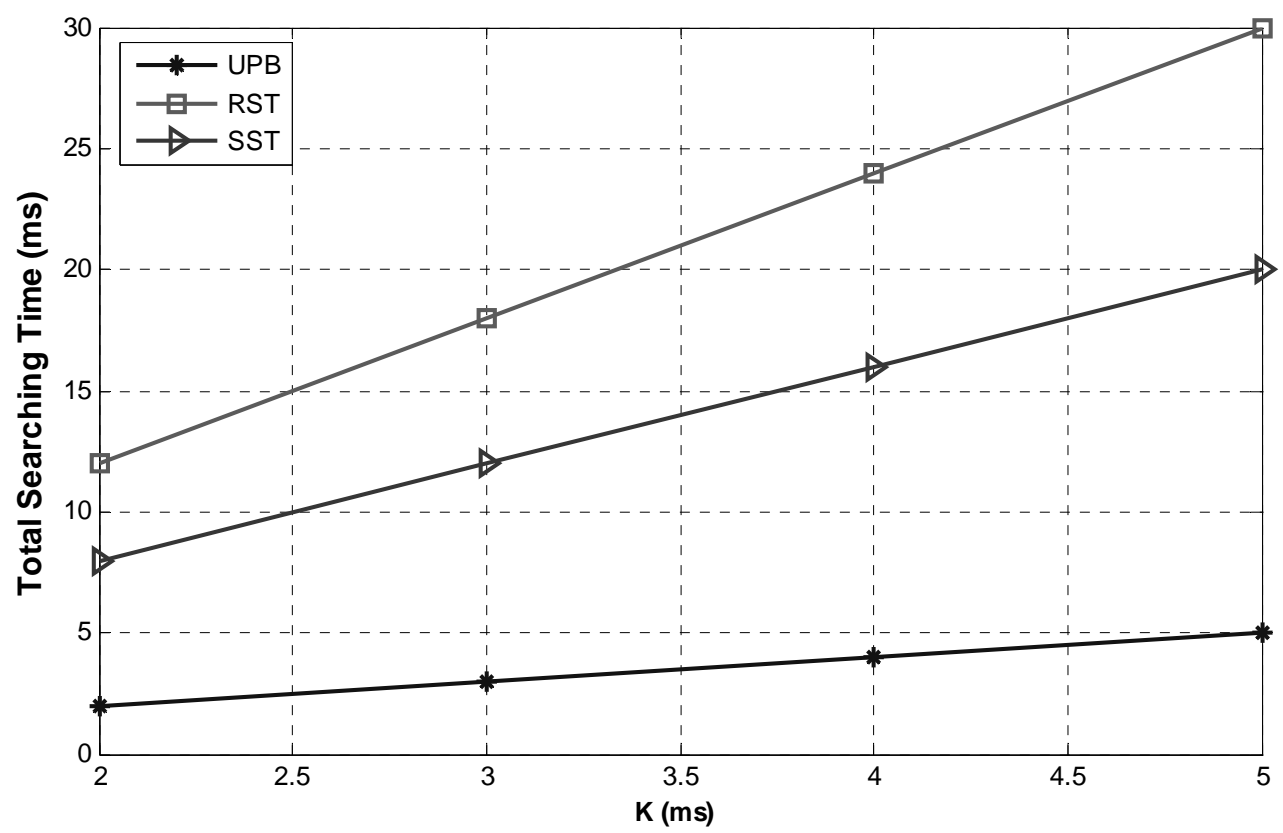

Figure 3. Total searching time vs. $K$. 
Average waiting time is also reduced in UPB scheme which is observed in Figure 4. We know that most likelihood location of user is cell 4 and we can search this location by using a UPB scheme in the first search. While if we search in any random order, then this location can be identified in search 1, 2, 3, 4, 5, 6, and 7. Increase in no. of searches will cause an increase in mean time spent by the user in the system. Hence we can say that

$$
\text { No. of search } \propto 1 / \mu_{r} \propto P_{\text {overload }}
$$

To conclude, it is established that any reduction in the number of searches will reduce overloading probability of VLR. Addition of extra VLR has been always a promising approach in location management [13]. The advantage of the proposed scheme is that it reduces the probability of overloading state in VLR and as soon as VLR will approach overloading, a certain number of users will be moving in $V L R_{w}$. To avoid any overloading in $V L R_{w}$, we have proposed to implement the UPB scheme which provides seamless service to mobile users even in case of VLR failure. Moreover the $V L R_{w}$ will play a vital role in such a scenario.

\section{Proposed Retrieval System Mechanism}

To understand the effectiveness of the proposed model, we assume that there is a single VLR in a network. Being a single VLR there is a higher probability of VLR overloading and heavy congestion. Location search in proposed $V L R_{w}$ is based on the UPB scheme which improves the performance of VLR. In case of overloading in main VLR, we have proposed to move a certain number of users in $V L R_{w}$. The Value of such users $\left(\right.$ User $\left._{\text {moves }}\right)$ will depend upon the threshold value defined by the operator. The probability of number of VLR user's movement in $V L R_{w}$ can be represented as:

$$
\text { User }_{\text {moves }}=\frac{1}{1-\left(P_{\text {failure }}+P_{\text {overload }}\right)}
$$

To improve the performance of VLR, we have taken a threshold value of 0.6 for $P_{\text {overload }}$ according to Figure 5. As $P_{\text {overload }} \geq 0.6$, Following no. of users should move in $V L R_{w}$ according equation to (2), shown in Figure 5.

Figure 5 indicates that, one has to move 2.8575 thousand users in $V L R_{w}$ to avoid overloading state of main VLR by using equation 3 . This method will reduce the Overloading state of original VLR. However, $V L R_{w}$ will come into the play only when overloading state is greater than or equal to 0.6 , but here the selection of users who will be allowed to move in $V L R_{w}$ will be a critical issue. As mentioned and established in the previous studies and proposed work that the performance of any location register including $V L R_{w}$ depends on searching capability to locate the called user. We have proposed to have user profile included in the $V L R_{w}$ database to employ the UPB scheme. This will give better results if the search is done according to maximum the probability to the minimum probability of steady state values of the user profile.

However, the criteria of user selection for $V L R_{w}$ can be viewed in terms of $4 \mathrm{G}$ systems which can provide a solution for selecting such users. The next section sug-

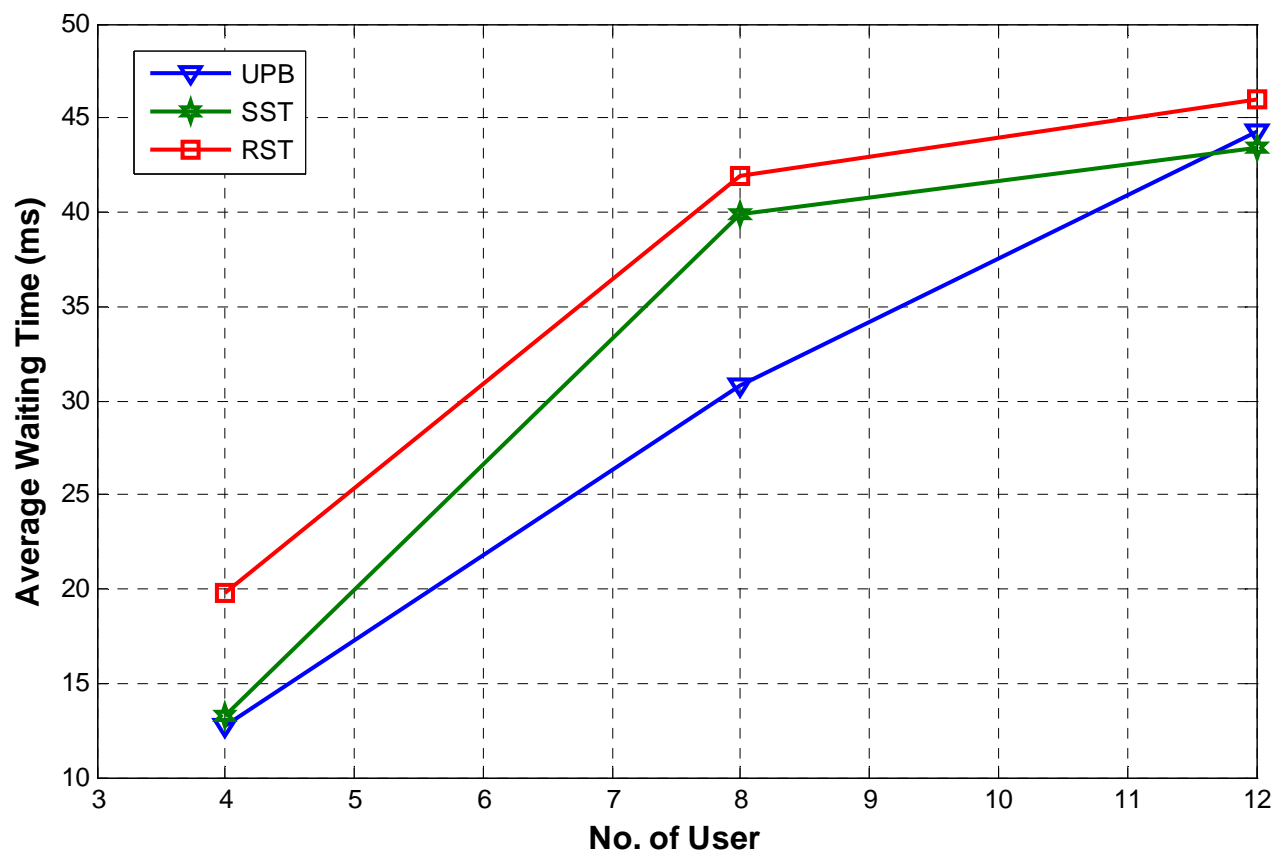

Figure 4. Comparison among UPB, SST and RST. 


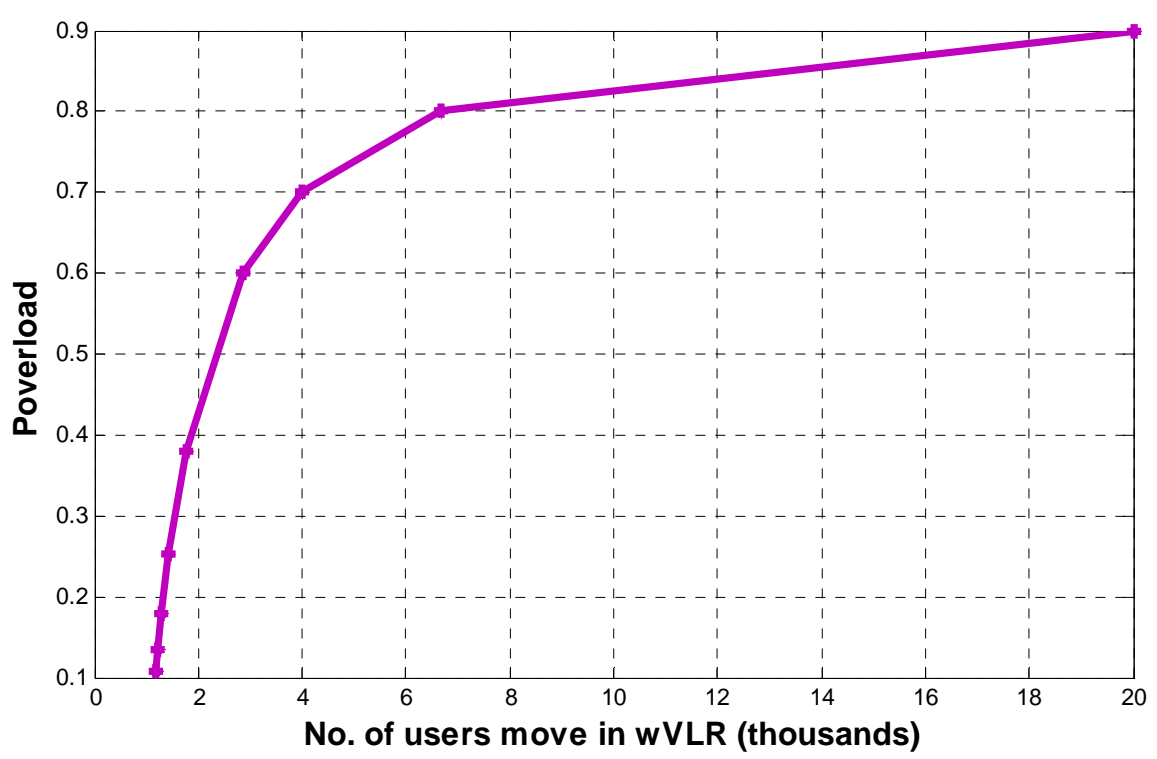

Figure 5. No. of user move in $V L R_{W}$.

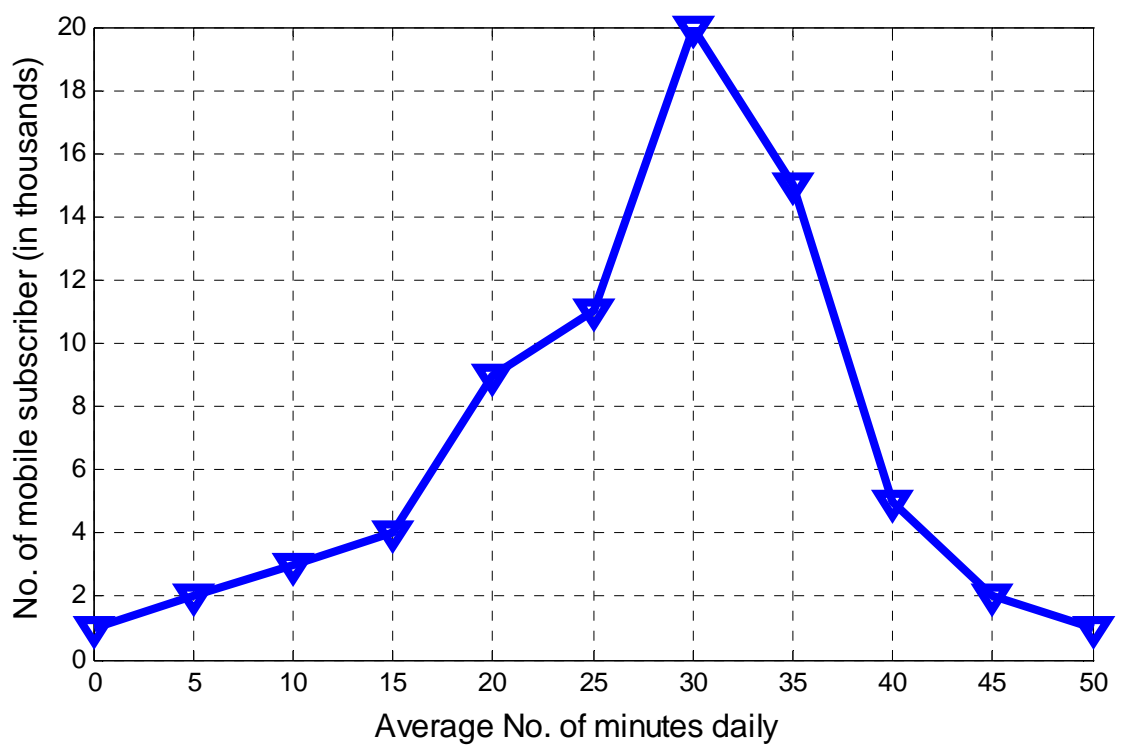

Figure 6. Selection of user on the basis of average no. of minutes spend daily.

gests the role of proposed work from the service point of view of $4 \mathrm{G}$ network which is mainly a personalized service network.

\section{Criteria of User Selection for Movement in $V L R_{w}$}

We can have various criteria of user selection for movement in $V L R_{w}$ to provide better quality of service and additional features for mobile subscribers. The attribute to identify valuable mobile subscribers can range from:

- Subscribers using the same number from last three years.

- Utilizing their services for 40 minutes or more on a daily basis.

- Utilizes M-banking and M-transaction to subscriber bills.

- Internet activity for surfing the internet or downloading over a GPRS connection

One can take any criteria to select the mobile subscriber to be moved in $V L R_{w}$ at the time of VLR overloading or failure. Here we have considered the average number of minutes spent by a user in the system. We have also assumed some statistics about average no. of minute's spent by mobile subscribers daily as shown in Figure 6.

We have taken on an average 45 minutes as a threshold value. This means that if a user is suffering from the 
overloading of main VLR, then the users fulfilling this criterion will be moved in $V L R_{w}$ for the service otherwise they have to wait in main VLR to get the service. According to Figure 6, three thousand users are fulfilling the criteria of the valuable mobile subscriber.

\section{Conclusion}

In this work we have introduced a user profile based (UPB) scheme based on the mobility information on a user. Further, UPB scheme was employed in a waiting VLR known as $V L R_{w}$ suggested to be used in shifting the excessive users from main VLR to avoid the probability of VLR overloading. Selection of users for shifting in $V L R_{w}$ is based on the average no. of minutes spent by them daily. It was established through simulation and analysis that the proposed UPB scheme is useful in tracking the called mobile user in minimum searches and will be effective in case of VLR failure or when there is a heavy congestion on the network. Applications of the proposed study for $4 \mathrm{G}$ network demonstrate the strength of this scheme. However, the performance of the work in terms of scalability to large numbers of users will be the limitation, but in context to 4G Network, where integration of heterogeneous network is a key issue. The proposed scheme can be useful for a specific home/organization/enterprise network.

\section{REFERENCES}

[1] Y.-B. Lin and I. Climatic, "Wireless and Mobile Network Architecture,” John Wiley \& Sons, New York, 2000.

[2] W. Stallings, "Wireless Communications and Networks," Prentice Hall, Upper Saddle River, 2001, pp. 291-297.

[3] A. Sehgal and R. Agrawal, "QoS Based Network Selection Scheme for 4G Systems," IEEE Transactions on Consumer Electronics, Vol. 56, No. 2, 2010, pp. 560-565. doi:10.1109/TCE.2010.5505970

[4] M. L. Roberts, et al., "Evolution of the Air Interface of
Cellular Communications Systems toward 4G Realization,” IEEE Communications Surveys and Tutorials, Vol. 8, No. 1, 2006, pp. 2-23. doi:10.1109/COMST.2006.323439

[5] H. Abu-Ghazaleh and A. S. Alfa, "Application of Mobility Prediction in Wireless Networks Using Markov Renewal Theory," IEEE Transactions on Vehicular Technology, Vol. 59, No. 2, 2010, pp. 788-802. doi:10.1109/TVT.2009.2037507

[6] M.-H. Jin, J.-T. Horng, M.-F. Tsai, E. H.-K. Wu and Eric H.-K. Wu, "Location Query Based on Moving Behaviors,” Information Systems, Vol. 32, No. 3, 2007, pp. 385401. doi:10.1016/j.is.2005.11.009

[7] J.-W. Lin and M.-F. Yang, "Dependable Public Wireless LANs without Hardware Support,” Fourth Annual ACIS International Conference on Computer and Information Science, Washington DC, 2005, pp. 628-633.

[8] M. H. Sun and D. M. Blough, "Mobility Prediction Using Future Knowledge," Proceedings of the 10th ACM Symposium on Modeling, Analysis, and Simulation of Wireless and Mobile Systems, New York, 2007, pp. 235-239.

[9] V. Bharghavan and M. Jayanth, "Profile Based Next-Cell Prediction in Indoor Wireless LAN," Proceedings of IEEE SICON’97, April 1997.

[10] R. Vaidya, C. Yadav, J. Kunkumath and P. Yadati, "Network Congestion Control: Mechanisms for Congestion Avoidance and Recovery," Proceedings of the 1st International Conference on Wireless Technologies for Humanitarian Relief, New York, 2011, pp. 199-207.

[11] K. S. Trivedi, "Probability \& Statistics with Reliability, Queuing and Computer Science Applications,” 8th Edition, John Wiley \& Sons, New York, 1999, pp. 309-359.

[12] Pushpa, A. Sehgal and R. Agrawal, "New Handover Scheme Based on User Profile: A Comparative Study," Second International Conference on Communication Software and Networks, 26-28 February 2010, pp. 18-21. doi:10.1109/ICCSN.2010.102

[13] J.-W. Lee, "Broadcasting Scheme for Location Management in Mobile Networks," International Journal of Software Engineering and Its Application, Vol. 2, No. 1, 2010, pp. 11-20. 